\title{
Modulators of microglia activation and polarization in ischemic stroke (Review)
}

\author{
CHENG-TING JIANG, WAN-FENG WU, YI-HUI DENG and JIN-WEN GE \\ Hunan Province Key Laboratory of Cerebrovascular Disease Prevention and \\ Treatment of Integrated Traditional Chinese and Western Medicine, College of Integrated Traditional Chinese \\ and Western Medicine, Hunan University of Chinese Medicine, Changsha, Hunan 410208, P.R. China
}

Received September 25, 2019; Accepted February 6, 2020

DOI: $10.3892 / \mathrm{mmr} .2020 .11003$

\begin{abstract}
Ischemic stroke is one of the leading causes of mortality and disability worldwide. However, there is a current lack of effective therapies available. As the resident macrophages of the brain, microglia can monitor the microenvironment and initiate immune responses. In response to various brain injuries, such as ischemic stroke, microglia are activated and polarized into the proinflammatory M1 phenotype or the anti-inflammatory M2 phenotype. The immunomodulatory molecules, such as cytokines and chemokines, generated by these microglia are closely associated with secondary brain damage or repair, respectively, following ischemic stroke. It has been shown that M1 microglia promote secondary brain damage, whilst M2 microglia facilitate recovery following stroke. In addition, autophagy is also reportedly involved in the pathology of ischemic stroke through regulating the activation and function of microglia. Therefore, this review aimed to provide a comprehensive overview of microglia activation, their functions and changes, and the modulators of these processes, including transcription factors, membrane receptors, ion channel proteins and genes, in ischemic stroke. The effects of autophagy on microglia polarization in ischemic stroke were also reviewed. Finally, future research areas of ischemic stroke and the implications of the current knowledge for the development of novel therapeutics for ischemic stroke were identified.
\end{abstract}

Correspondence to: Professor Yi-Hui Deng or Professor Jin-Wen Ge, Hunan Province Key Laboratory of Cerebrovascular Disease Prevention and Treatment of Integrated Traditional Chinese and Western Medicine, College of Integrated Traditional Chinese and Western Medicine, Hunan University of Chinese Medicine, 300 Xueshi Road, Hanpu Science and Education Park, Yuelu, Changsha, Hunan 410208, P.R. China

E-mail: 644138330@qq.com

E-mail: 40831556@qq.com

Key words: microglia, ischemic stroke, polarization, autophagy, immune responses, pathology, modulation mechanisms

\section{Contents}

1. Introduction

2. Microglia

3. Modulatory mechanisms of microglia polarization

4. Autophagy

5. Conclusions

\section{Introduction}

According to a recent report from the World Health Organization, ischemic stroke is the second leading cause of mortality worldwide (1). In 2016 alone, 5.5 million people succumbed to an ischemic stroke and an estimated 116.4 million people suffered from permanent disabilities following a stroke event worldwide (2). Undoubtedly, these mortalities and disabilities have a huge effect, both emotionally and financially, on families and society.

Ischemic stroke is primarily caused by the blockage of cerebrovascular blood flow. Previous studies have reported that the incidence of early mortality following ischemic stroke (between 21 days to 1 month) in both high-income and low-middle-income countries increased gradually from 2000 to 2008 , from 13 to $23 \%$ and 13 to $19 \%$, respectively $(3,4)$. Currently, intravenous thrombolysis and mechanical thrombectomy are the only effective means of treating ischemic stroke $(5,6)$; however, these treatment options are limited by the short-recommended treatment window (6). In addition, a series of reperfusion injuries may occur following ischemic reperfusion $(6,7)$; in particular, inflammation and oxidative stress are the main causes of reperfusion injuries, and the latter can induce the former (6-9). An increasing amount of evidence has demonstrated that continuous neuroinflammation can damage neurons and the blood-brain barrier (BBB) during the process of cerebral infarction, and this in turn results in tissue destruction and worsens the functional outcome (10-12). In addition, it has been demonstrated that the inhibition of neuroinflammation decreased the neurological deficit in experimental stroke models $(12,13)$. Therefore, it is evident that neuroinflammation serves a crucial role in ischemic stroke-induced brain injury.

Microglia are important cells that initiate the inflammatory response in the brain following various brain injuries (14), 
including ischemic stroke (15). Accumulating evidence has indicated that activated microglia are the primary source of immunomodulatory molecules, such as cytokines, chemokines and free radicals, in the brain (16-18); these molecules are closely associated with secondary brain injury and brain tissue repair in ischemic stroke (16-18). In addition, some molecules, including soluble Fas ligand (19) and endothelial nitric oxide synthase $(20,21)$, which are generated by other cells, such as neurons and endothelial cells, have also been identified to affect the stroke outcome through interacting with microglia in the brain following ischemic stroke (19). Therefore, investigating microglial changes and their function is crucial to understanding ischemic stroke pathophysiology.

To develop new and effective therapies for ischemic stroke, it is also necessary to investigate the cellular and molecular mechanisms underlying brain damage and tissue repair in ischemic stroke. Despite the fact that microglia-induced inflammation can aggravate brain damage upon ischemic stroke, the modulation of the microglial phenotype has also been observed to promote brain repair; for example, through decreasing brain edema, improving white matter integrity and recovering motor function (13). Therefore, investigating the mechanisms underlying microglial phenotypic transformation is crucial for the development of novel treatments for patients with ischemic stroke.

The present review aimed to describe the important advances in the current understanding of the changes, functions and modulators of microglia during ischemic stroke. The roles of autophagy in the modulation of microglia polarization are discussed, in addition to the future research directions of ischemic stroke, which may help identify novel treatment strategies for ischemic stroke.

\section{Microglia}

Microglia are small, macrophage-like glial cells that account for $10-15 \%$ of cells in the central nervous system (CNS). In response to various stresses, microglia are rapidly activated to differentiate into either the M1 or the M2 phenotype, which are involved in tissue damage and repair, respectively (22-24).

M1 microglia and changes. M1 microglia exist in a proinflammatory state and secrete proinflammatory cytokines, which have been identified to promote brain damage $(13,19)$. Following ischemic stroke, M1 microglia are activated and subsequently serve a detrimental role. Briefly, upon experiencing ischemia/hypoxia, $\mathrm{NF}-\kappa \mathrm{B}$ is activated within microglia and translocates from the cytoplasm to the nucleus; this activates the release of proinflammatory cytokines, which causes secondary brain damage (16-18), such as interleukin (IL)-1 $\beta$ (24), IL-6 (25) and tumor necrosis factor- $\alpha$ (TNF- $\alpha$ ) (26), in addition to the production of inducible nitric oxide synthase (iNOS) $(27,28)$. For example, in middle cerebral artery occlusion (MCAO) model mice, TNF- $\alpha$ secreted by M1 microglia was identified to increase endothelial necroptosis and BBB leakage following ischemic stroke $(21,29)$. This further promotes neuroinflammation and brain edema, resulting in a poor outcome (29). Moraes et al (30) demonstrated that IL-1 $\beta$, which is generated by M1 microglia, decreased the number of synapses in the hippocampus and cortex, which aggravated cognitive impairment. In oxygen-glucose-deprived (OGD) microglia, the increase in IL-17A levels aggravated neuronal death (31). In addition, C-C motif chemokine ligand 2 (CCL2) generated by microglia was observed to recruit $\mathrm{CD} 8^{+} \gamma \delta \mathrm{T}$ cells to the ischemic brain, which was identified to be the primary factor aggravating brain infarction $(32,33)$. In fact, besides ischemic stroke, M1 microglia activation occurs in multiple other neurological diseases; for example, in an RD1 mouse model of retinal degeneration, flow cytometry analysis revealed that the percentage of T-lymphocyte activation antigen CD86 (CD86) ${ }^{+}$ M1 microglia was markedly increased in the rapid degeneration phase (34); paraquat stimulation was revealed to activate M1 microglia, which subsequently activated the toll-like receptor-4 (TLR4)-mediated NF- $\kappa B$ signaling pathway and resulted in the release of proinflammatory cytokines (35); and the number of low affinity immunoglobulin gamma $\mathrm{Fc}$ region receptor III-A/b (CD16/32) ${ }^{+}$M1 microglia was significantly increased in the brains of subarachnoid hemorrhage model mice through the activation of the myeloid differentiation primary response protein MyD88 (MyD88)/mitogen-activated protein kinase pathway, whereas decreasing the percentage of M1 microglia significantly improved the neurological deficits (36). Other results from the literature have indicated that M1 microglia activation can also aggravate brain damage in other types of neurological disease (34-36).

The activation of M1 microglia can be determined by detecting the expression of their surface markers (Table I); these classical markers include integrin alpha-M (CD11b) (37), CD16 (38), CD32 (39) and CD86 (40). However, these markers cannot distinguish between microglia and macrophages in the brain, as they can be expressed by both M1 microglia and macrophages. For example, Liu et al (41) reported that both $\mathrm{CD}^{+} 6^{+}$/ionized calcium-binding adapter molecule $1(\mathrm{Iba1})^{+} \mathrm{M} 1$ microglia/macrophages were detected using double immunofluorescence staining. To distinguish resident microglia from blood-derived macrophages in the brain, Satoh et al (42) suggested that TMEM119 may be used as a marker of resting microglia in the human brain; however, the challenge remains to discriminate activated microglia from infiltrated macrophages in the brain.

Changes in the activation of M1 microglia can affect stroke prognosis and changes in the expression levels of M1 microglia biomarkers, including surface markers and proinflammatory cytokines, are also common in ischemic stroke $(13,25,43)$. Based on these changes, it is possible to draw some useful conclusions regarding ischemic stroke. For example, in MCAO mice, the expression levels of certain proinflammatory cytokines, such as IL-1 $\beta$, TNF- $\alpha$ and IL- 6 , in addition to iNOS, were observed to increase from $12 \mathrm{~h}$, peak at day 14 , then subsequently decrease and remain constant in the ischemic boundary zone until day 28 following MCAO $(25,43)$. In addition, the study (25) also observed that CD32 expression levels increased in a similar manner. Another previous study drew similar conclusions, witnessing a significant increase in CD16 expression levels from day 3 , which remained elevated until day 14 in the cortex and striatum at the inner boundary of the infarct (44). These conclusions were further confirmed in both a bilateral common carotid artery ligation (BCCA) model and a photothrombotic (PT) stroke model. In the hippocampal CA1 region of the BCCA ligation mice, the M1 microglia marker CD74 was observed to be expressed from day 3 post-ischemic 
Table I. Markers of M1 and M2 microglia.

\begin{tabular}{|c|c|c|c|c|c|}
\hline Phenotype & Marker & Type & Effect & (Refs.) & Author, year \\
\hline \multirow[t]{17}{*}{ M1 } & IL-1 $\beta$ & Cytokine & Proinflammatory & $(25)$ & Zhu et al, 2019 \\
\hline & IL-6 & Cytokine & Proinflammatory & $(59)$ & Shu et al, 2016 \\
\hline & TNF- $\alpha$ & Cytokine & Proinflammatory & $(25)$ & Zhu et al, 2019 \\
\hline & iNOS & Metabolic enzyme & Oxidative damage & $(59)$ & Shu et al, 2016 \\
\hline & ROS & Metabolite & Oxidative damage & $(7)$ & $\mathrm{Xu}$ et al, 2018 \\
\hline & CD11b & receptor & Induce proinflammatory signaling & $(37,81)$ & $\begin{array}{l}\text { Liu et al, 2018; } \\
\text { Hyakkoku et al, } 2010\end{array}$ \\
\hline & CD16 & Receptor & Induce proinflammatory signaling & $(38)$ & Jiang et al, 2018 \\
\hline & CD32 & Receptor & Induce proinflammatory signaling & $(37)$ & Liu et al, 2018 \\
\hline & CD68 & Receptor & Induce proinflammatory signaling & $(27)$ & Boddaert et al, 2018 \\
\hline & CD86 & Receptor & Induce proinflammatory signaling & $(44)$ & Hu et al, 2012 \\
\hline & NF-KB & Transcription & $\begin{array}{l}\text { Induce M1 phenotype, elevate the } \\
\text { expression of proinflammatory cytokines }\end{array}$ & $(16)$ & Yang et al, 2019 \\
\hline & STAT1 & Transcription & Elevate the expression of $\mathrm{NF}-\kappa \mathrm{B}$ & $(70)$ & Butturini et al, 2019 \\
\hline & STAT3 & Transcription & Elevate the expression of NF- $\kappa \mathrm{B}$ & $(72)$ & Ding et al, 2019 \\
\hline & Hv1 & Ion channel protein & $\begin{array}{l}\text { Elevate the expression of ROS and } \\
\mathrm{NF}-\kappa \mathrm{B} \text {, induce } \mathrm{M} 1 \text { phenotype }\end{array}$ & $(94,96)$ & $\begin{array}{l}\text { Tian et al, 2016; } \\
\text { Yu et al, } 2018\end{array}$ \\
\hline & Kv1.3 & Ion channel protein & $\begin{array}{l}\text { Proinflammatory, induce M1 } \\
\text { phenotype }\end{array}$ & $(83,97)$ & $\begin{array}{l}\text { Di Lucente et al, 2018; } \\
\text { Nguyen et al, } 2017\end{array}$ \\
\hline & H19 & Gene & $\begin{array}{l}\text { Proinflammatory, induce M1 } \\
\text { phenotype }\end{array}$ & $(98)$ & Wang et al, 2017 \\
\hline & miRNA-155 & Gene & $\begin{array}{l}\text { Proinflammatory, induce M1 } \\
\text { phenotype }\end{array}$ & $(18)$ & Zheng et al, 2018 \\
\hline \multirow[t]{12}{*}{ M2 } & IL-4 & Cytokine & $\begin{array}{l}\text { Anti-inflammatory, induce M2 } \\
\text { phenotype }\end{array}$ & $(23)$ & Liu et al, 2016 \\
\hline & IL-10 & Cytokine & Anti-inflammatory & $(25)$ & Zhu et al, 2019 \\
\hline & TGF- $\beta$ & Cytokine & Anti-inflammatory, regeneration & $(44)$ & Hu et al, 2012 \\
\hline & $\mathrm{Ym} 1 / 2$ & Protein & Anti-inflammatory, regeneration & $(25)$ & Zhu et al, 2019 \\
\hline & Arg-1 & Protein & Anti-inflammatory, regeneration & $(44)$ & Hu et al, 2012 \\
\hline & CCL22 & Chemokine & Recruits regulatory $\mathrm{T}$ cells & $(44)$ & Hu et al, 2012 \\
\hline & CD206 & Receptor & Induce anti-inflammatory signaling & (39) & Jin et al, 2014 \\
\hline & STAT6 & Transcription & Inhibit the expression of $\mathrm{NF}-\kappa \mathrm{B}$ & (74) & Yang et al, 2017 \\
\hline & Nrf2 & Transcription & Anti-inflammatory, antioxidant stress & $(7)$ & $\mathrm{Xu}$ et al, 2018 \\
\hline & $\operatorname{PPAR} \gamma$ & Transcription & $\begin{array}{l}\text { Inhibit the expression of } \mathrm{NF}-\kappa \mathrm{B} \text {, } \\
\text { promote the expression of } \mathrm{Nrf} 2\end{array}$ & $(26,78)$ & $\begin{array}{l}\text { Liu et al, 2018; } \\
\text { Cai et al, } 2017\end{array}$ \\
\hline & miRNA-124 & Gene & $\begin{array}{l}\text { Anti-inflammatory, induce M2 } \\
\text { phenotype }\end{array}$ & (99) & Hamzei et al, 2016 \\
\hline & FAM19A3 & Gene & $\begin{array}{l}\text { Anti-inflammatory, induce M2 } \\
\text { phenotype }\end{array}$ & $(100)$ & Shao et al, 2015 \\
\hline
\end{tabular}

IL, interleukin; TNF- $\alpha$, tumor necrosis factor- $\alpha$; iNOS, inducible nitric oxide synthase; miRNA, microRNA; ROS, reactive oxygen species; CD11b, integrin alpha-M; CD16, low affinity immunoglobulin gamma Fc region receptor III-A; CD32, low affinity immunoglobulin gamma Fc region receptor III-b; CD68, cluster of differentiation 68; CD86, T-lymphocyte activation antigen CD86; CD206, macrophage mannose receptor 1; STAT, signal transducer and activator of transcription; H19, H19 imprinted maternally expressed transcript; TGF- $\beta$, transforming growth factor- $\beta$; Ym1/2, chitinase-3-like protein 3; Arg-1, arginase-1; CCL22, C-C motif chemokine ligand 22; Nrf2, nuclear factor erythroid 2-related factor 2; PPAR $\gamma$, Peroxisome proliferator-activated receptor $\gamma$; FAM19A3, chemokine-like protein TAFA-3.

insult and elevated from day 5 post-ischemia (45). Additionally, CD16 and iNOS expression levels were identified to be increased in infarct regions at day 42 following BCCA (46). In PT stroke model mice, CD16 and CD32 expression levels were detected at day 1 and remained elevated until day 14 following
PT cortical ischemia (47). In addition, numerous inflammatory cytokines, such as TNF- $\alpha$, IL-1 $\beta$ and IL-6, remained highly expressed at days 14 and 21 in the ischemic region following a PT stroke $(48,49)$. Other associated studies reported that age was also associated with microglia polarization, and the trend 
of the expression levels of M1 microglial markers in ischemic stroke was consistent with the former studies $(25,44)$ in both young adult and older mice $(50,51)$.

Based on these changes, it is suggested that the proinflammatory state may be dominant in the acute stage of ischemic stroke; however, the changes in the expression levels of proinflammatory cytokines during the chronic phase of ischemic stroke, and the interactions between them and brain tissue repair, remain unclear.

M2 microglia and changes. M2 microglia exist in an anti-inflammatory state and secrete anti-inflammatory cytokines and neurotrophic factors to promote brain repair $(23,25,52)$. M2 microglia are activated and reported to serve a beneficial role following ischemic stroke; for example, in conditions of ischemia/hypoxia, peroxisome proliferator-activated receptor $\gamma$ (PPAR $\gamma$ ), a transcription factor with anti-inflammatory properties, is reportedly activated and mobilized from the nucleus to the cytoplasm in microglia (26). This leads to the activation of M2 microglia, which release anti-inflammatory cy tokines that improve stroke outcome. For example, Liu et al (23) demonstrated that IL-4 secreted by M2 microglia decreased the infarct size following ischemic stroke and improved the long-term functional recovery. Zhu et al (25) observed that M2 microglia-induced chitinase-3-like protein 3 (Ym1/2), IL-10 and transforming growth factor- $\beta$ (TGF- $\beta$ ) secretion promoted angiogenesis, and thereby decreased the BBB leakage and improved stroke outcome. In addition, Choi et al (53) confirmed that M2 microglia promoted the proliferation and neuronal differentiation of neural stem/progenitor cells in the ipsilateral subventricular zone following ischemic stroke through upregulating the expression levels of TGF- $\alpha$; this may provide an effective therapy for neurogenesis. Additionally, macrophages recruited by microglia were identified to enhance M2 microglia polarization and improve stroke outcome $(54,55)$. Similar to M1 microglia, besides from ischemic stroke, M2 microglia activation occurs in numerous other types of neurological disease; for example, in both spinal cord injury and intracerebral hemorrhage mouse models, M2 microglia were identified to be activated, which released anti-inflammatory cytokines downstream of cAMP response element-binding protein (CREB)-associated signaling pathways $(56,57)$. In neonatal germinal matrix hemorrhage rats, Rh-Chemerin promoted the accumulation and proliferation of M2 microglia in periventricular regions and suppressed the inflammatory response through nuclear factor erythroid 2-related factor 2 (Nrf2)-associated signaling pathways (58). Previous studies demonstrated that M2 microglia activation can also promote brain tissue repair in other neurological diseases (56-58).

The activation of M2 microglia can be assessed by determining the expression levels of their surface markers (Table I), for example, macrophage mannose receptor 1 (CD206) (59), and the secretion of anti-inflammatory cytokines, including IL-4 (23), IL-10 (59), arginase-1 (Arg-1) (60), Ym1 (28) and TGF- $\beta$ (59). Changes in the percentage of activated M2 microglia can affect stroke prognosis and based on these changes, it is possible to draw some useful conclusions regarding ischemic stroke; for example, in MCAO model mice (44), the mRNA expression levels of cytokines generated by M2 microglia, including Arg-1, Yml/2, CCL22, IL-10 and $T G F-\beta$, were detected from days 1 to 3 following MCAO and peaked at days 3 to 5 post-injury in ischemic regions (44). The expression levels of the majority of M2 microglia genes was observed to begin to decrease at days 5-7 after MCAO and by day 14, were restored to pre-injury levels in ischemic regions (44). In addition, the number of CD206 ${ }^{+}$cells followed a similar trend (44); CD206 remained highly expressed until day 21 in the cortex and striatum following ischemia (61). Previous studies have further validated these results in a PT stroke model (47-49); in the ischemic cortex, CD206 expression levels rapidly increased at day 1, peaked at day 7 (47) and remained highly expressed until day 21 following a PT stroke (49). In addition, certain anti-inflammatory cytokines were also observed to be highly expressed until days 14 and 21 following a PT stroke; for example, IL-4 and IL-10 remained highly expressed until day 14 (47) and TGF- $\beta$ remained highly expressed until day 21 in ischemic regions (49). Furthermore, another previous study (44) revealed that the M2 phenotype was the dominant phenotype for the first 7 days following ischemic stroke and could promote neuronal survival following OGD.

Based on these data regarding the changes in the expression levels of M1 and M2 markers, several conclusions were drawn (Fig. 1); it was hypothesized that microglia are activated following ischemic stroke and the M1/M2-type pattern of expression changes dynamically post-injury, exhibiting an early beneficial M2 phenotype, followed by the transition to a detrimental M1 phenotype at the later stages. These results suggested that regulating microglia transformation from the M1 to M2 phenotype may be a crucial method of treating ischemic stroke.

\section{Modulatory mechanisms of microglia polarization}

It has been suggested that ischemic stroke may be effectively treated through modulating microglia polarization; in general, these modulators include four categories: Transcription factors; receptors; ion channels; and gene modulators.

Transcription factors. Transcription factors are proteins that bind to DNA and regulate the transcriptional activity of genes. $\mathrm{NF}-\kappa \mathrm{B}$, members of the STAT family, nuclear factor erythroid 2-related factor 2 (Nrf2) and PPAR $\gamma$ are 4 important transcription factors that have been associated with the mechanisms underlying microglia polarization $(16,19,26,62,63)$ (Fig. 2). The first 2 transcription factors are closely associated with M1 polarization $(16,19,62)$, whereas the other 2 are associated with M2 polarization $(26,63)$.

$N F-\kappa B$. The transcription factor $\mathrm{NF}-\kappa \mathrm{B}$ has been identified to activate microglia, and to transform these activated cells into the M1 phenotype $(16,62)$. In fact, it has been confirmed that NF- $\kappa \mathrm{B}$ can be activated in response to detrimental stresses and induce inflammatory responses in the brain $(16,62)$. Upon ischemia or hypoxia, the expression levels of some damage-associated molecules, including protein S100-B $(11,64)$, chemokine-like factor 1 (65-67) and CXC motif ligand 12 (43) are reportedly increased; these molecules bind to membrane receptors, such as receptor for advanced glycation end products, C-C 


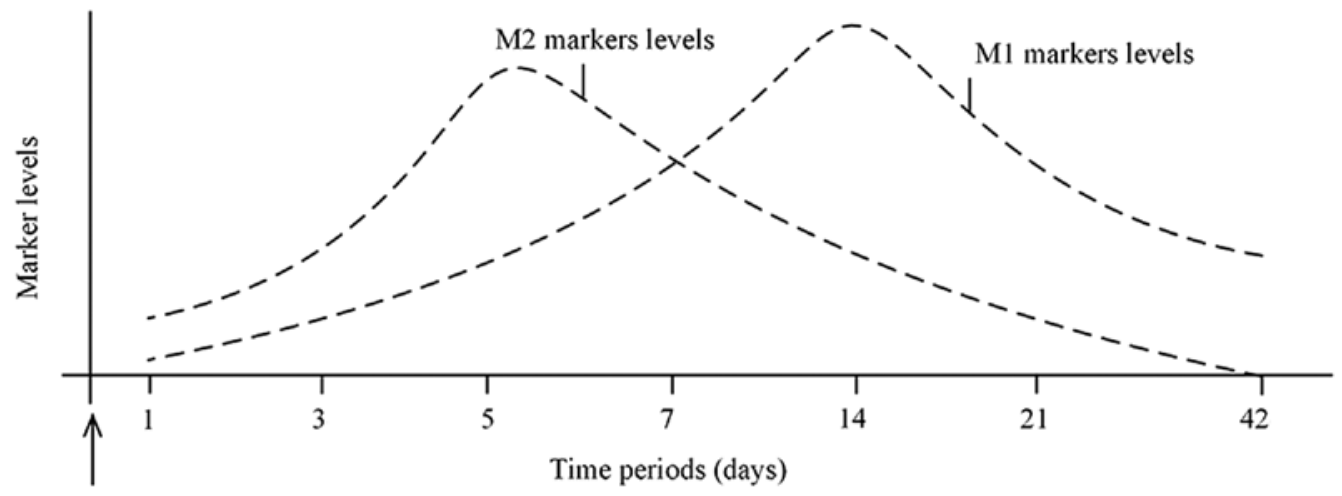

IS induction

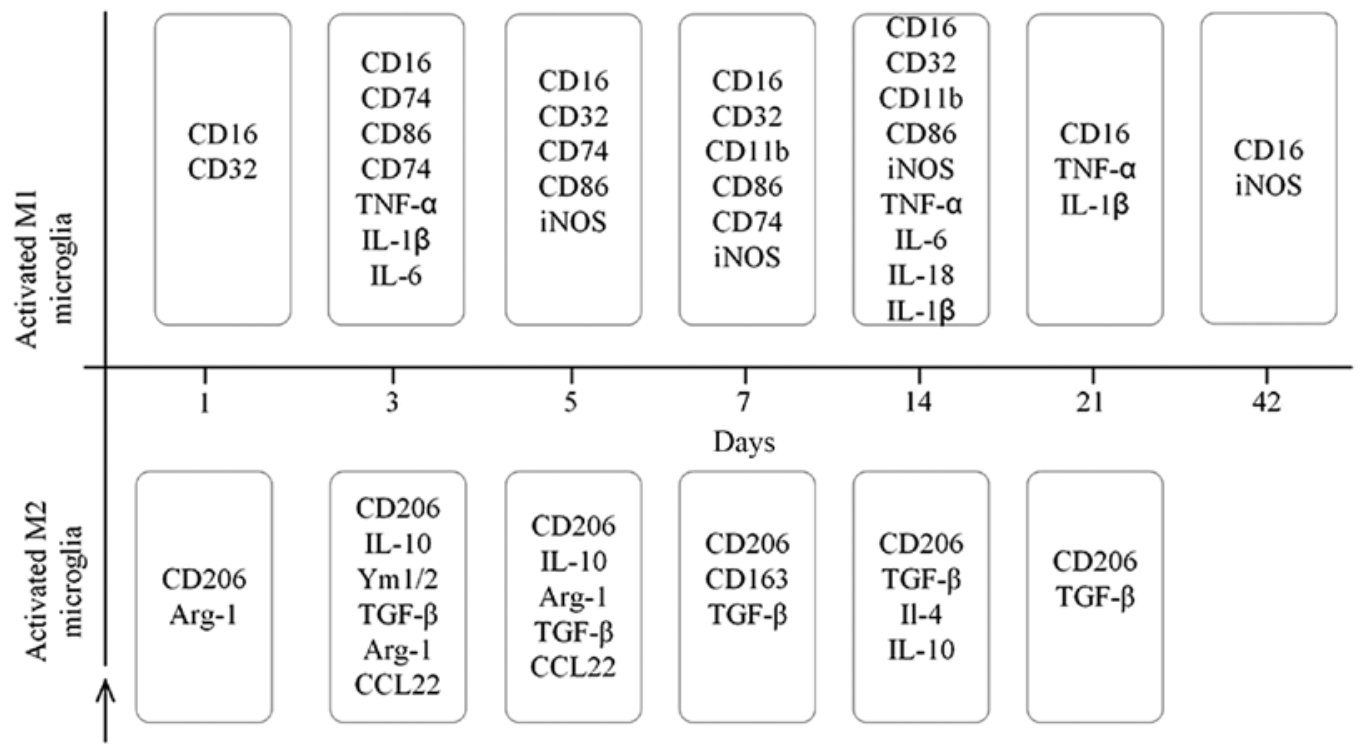

IS induction

Figure 1. Dynamic changes in microglia marker levels over time following IS. Top panel: M1 microglia markers demonstrated an increasing trend during the first 14 days following IS, after which they decreased. Expression levels of M2 microglia markers increased from day 1, peaked at days 5-7 and decreased until day 42. Bottom panel: Microglia exhibited an M2-like response as early as 1 day following IS, which manifested as increased expression levels of CD206 Arg-1. The expression levels of TGF- $\beta$ increased from day 3 until day 21 . With regards to the M1 markers, the expression levels of the proinflammatory cytokines, TNF- $\alpha$, IL- 6 and IL-1 $\beta$ increased from day 3. The levels of M1 surface markers CD16 and CD32 increased from day 1 and CD16 and iNOS expression levels remained increased until day 42 after IS. IS, ischemic stroke; Arg-1, arginase 1; TGF- $\beta$, transforming growth factor- $\beta$; TNF- $\alpha$, tumor necrosis factor $\alpha$; IL, interleukin; iNOS, inducible nitric oxide synthase; CCL, C-C motif chemokine ligand; Ym1/2, chitinase-3-like protein 3.

chemokine receptor 4 and $\mathrm{CXC}$-chemokine receptor 4 to activate $\mathrm{NF}-\kappa \mathrm{B}(43,64-66)$. The activated $\mathrm{NF}-\kappa \mathrm{B}$ is subsequently translocated to the nucleus, where it triggers inflammatory responses (16). NF- $\kappa \mathrm{B}$ inhibitor $\alpha(\mathrm{I} \kappa \mathrm{B} \alpha)$, a protein bound to $\mathrm{NF}-\kappa \mathrm{B}$, can inhibit the activation of NF- $\kappa \mathrm{B}$; however, upon phosphorylation, it can release $\mathrm{NF}-\kappa \mathrm{B}$, which translocates to the nucleus from the cytoplasm $(16,68)$. The activated $N F-\kappa B$ can promote the secretion of proinflammatory cytokines from M1 microglia, such as IL-18, IL-6 and TNF- $\alpha$ (16). For example, in MCAO model mice, a significant increase in $\mathrm{NF}-\kappa \mathrm{B}(\mathrm{p} 65)$ expression levels were detected in the ischemic brain, which was accompanied by an increase in IL-18, IL-6 and TNF- $\alpha$ expression levels, whereas an inhibitor of NF- $\kappa \mathrm{B}$ significantly inhibited the release of these cytokines (16). In addition, inhibiting the increased upregulation of activated $\mathrm{NF}-\kappa \mathrm{B}(\mathrm{p} 65)$ decreased the production of hypoxia-induced factor- $1 \alpha$, which then attenuated the neuroinflammatory responses in ischemic stroke (69).
STAT family members. STAT1-STAT6 exist in the cytoplasm and serve an important role in the regulation of inflammatory responses. In hypoxia-activated BV2 microglia, the phosphorylation levels of STAT1 were significantly increased, which was accompanied by increased expression levels of M1 microglia markers, such as cluster of differentiation 68 and iNOS (70); however, the expression levels of these markers were significantly decreased in STAT1 ${ }^{-/-}$BV2 cells following hypoxia. Several previous studies have also identified that STAT3 is also associated with the regulation of M1 microglia polarization in both an MCAO-induced and a bilateral common carotid arteries stenosis (BCAS)-induced model of ischemic stroke (19,71-73). Ding et al (72) identified that the inhibition of the Janus kinase 2 (JAK2)/STAT3 signaling pathway promoted the transition from resting microglia to the M2 phenotype to exert anti-inflammatory effects; briefly, the phosphorylation levels of JAK 2 and STAT3 were increased in the acute phase of cerebral ischemia in the mice, which was 


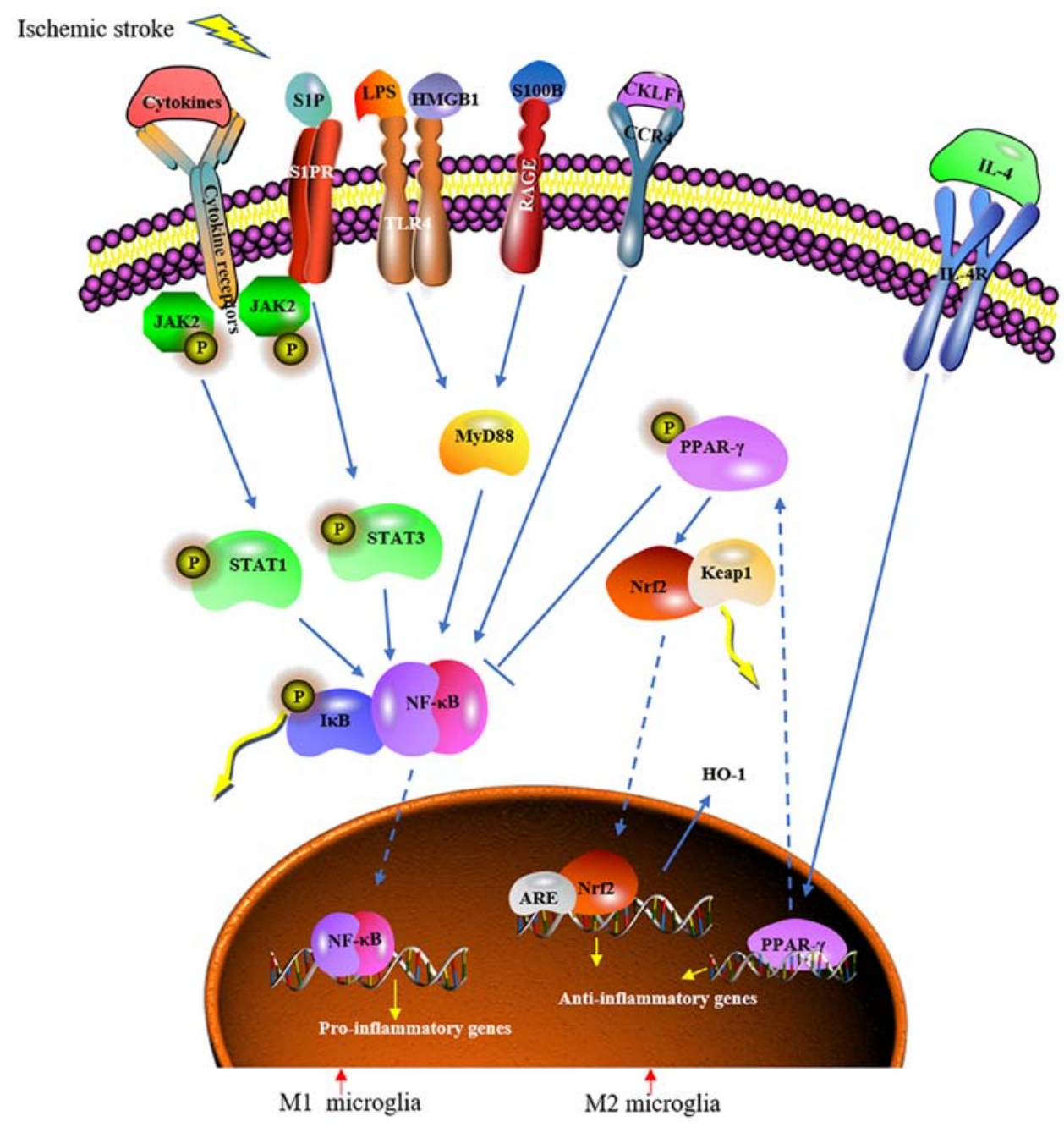

Figure 2. Modulatory mechanisms of microglia polarization following ischemic stroke. Microglia are activated following ischemic stroke. M1 microglia activation involves different factors, including the cytokines S1P, LPS, HMGB1, S100B and CKLF1. These factors bind to membrane receptors, such as the cytokine receptors S1PR, RAGE, TLR4, IL-4R and CCR4, to trigger proinflammatory cellular signaling pathways. These pathways include the JAK2/STAT1/NF-KB,

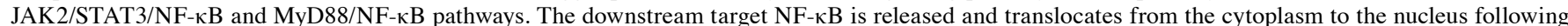
the phosphorylation of IKB, where it initiates the transcription of proinflammatory genes. M2 microglia can be activated by IL-4, which promotes PPAR $\gamma$ mobilization from the nucleus to the cytoplasm. Subsequently, PPAR $\gamma$ inhibits the activation of NF- $\mathrm{KB}$ and promotes the activation of Nrf2 to induce the transcription of anti-inflammatory genes. Additionally, the activated Nrf2 promotes the expression of HO-1, which scavenges reactive oxygen species and nitric oxide. S1P, sphingosine 1 phosphate; LPS, lipopolysaccharide; IL, interleukin; HMGB1, high mobility group protein B1; S100B, protein S100-B; CKLF1, chemokine-like factor; SIPR, sphingosine 1 phosphate receptor; TRL4, toll-like receptor 4; CCR4, C-C chemokine receptor type 4; RAGE; JAK2, Janus acti-

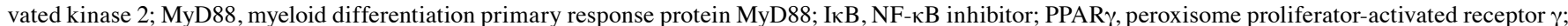
$\mathrm{Nrf2}$, nuclear factor erythroid 2-related factor 2; HO-1, heme oxygenase 1; Keap 1, Kelch-like ECH-associated protein 1; STAT, signal transducer and activator of transcription.

accompanied by a significant increase in M1 microglia markers, including CD16, IL-1 $\beta$ and TNF- $\alpha$, and a mild increase in M2 microglia markers, such as CD206 and IL-10. However, SC99, an inhibitor targeting the JAK2/STAT3 signaling pathway, inhibited the production of M1 markers and increased the expression of M2 markers, exerting significant anti-inflammatory effects both in vivo and in vitro. Similarly, another study demonstrated that AG490 (a tyrosine kinase inhibitor of JAK2) decreased the expression levels of M1 markers, iNOS, IL- $1 \beta$, TNF- $\alpha$, CD16 and CD32, the phosphorylation of I $\mathrm{KB} \alpha$ and translocation of p50/p65 in serum soluble tumor necrosis factor ligand superfamily member 6 (Fas ligand)-induced M1 microglia (19). Conversely, another previous study demonstrated that the activated STAT3 and STAT6 pathways could promote the polarization of nonactivated microglia to the M2 phenotype (74). Therefore, based on these contradictory observations, further studies are required to elucidate whether STATs and their associated signaling pathways can regulate microglia polarization.

$N r f 2$. The redox-sensitive transcription factor Nrf2 demonstrates both antioxidant and anti-inflammatory properties (75,76), and $\mathrm{Nrf} 2$ and its associated signaling pathways have been identified to exert a protective effect following ischemic stroke; for example, in $\mathrm{Nrf2}^{-\digamma}$ mice, the neurological deficit and cerebral infarct volume were both aggravated following MCAO compared with the wild-type mice (75). Meanwhile, the beneficial effects of sirtuin 6 , a member of the sirtuin family of $\mathrm{NAD}^{+}$-dependent deacetylases, were abolished in $\mathrm{Nrf}^{-/-}$mice following ischemic stroke, in that the reactive oxygen species (ROS) levels and the number of dead neuro-2A cells in $\mathrm{Nrf}^{-/-}$mice were increased (75). In addition, another 
previous study demonstrated that L-F001, a novel multifunctional Rho-associated protein kinase inhibitor, increased the expression levels of the M2 microglia marker CD206 through the activation of Nrf2 signaling pathways in vitro (63). These beneficial results have also been validated in vitro; in oxygen-glucose deprivation/reperfusion (OGDR)-activated BV2 microglia, Nrf2 was observed to be activated and dissociated from the repressor protein, Kelch ECH-associating protein 1. This facilitated its translocation from the cytoplasm to the nucleus, where it bound to the antioxidant response element (ARE) and induced the expression of downstream genes, such as NADPH quinone oxidoreductase and heme oxygenase 1, which scavenge ROS and nitric oxide $(7,68,77)$. Additionally, the activation of Nrf2 was identified to decrease ROS, NOD like receptor family pyrin domain containing 3 (NLRP3) and IL-1 $\beta$ expression levels in BV2 microglia (7). However, despite the evident protective role of $\mathrm{Nrf2}$, further studies are required to elucidate whether this transcription factor and its signaling pathways may regulate M2 microglia polarization and function following ischemic stroke.

PPAR $\gamma$. PPAR $\gamma$ is a ligand-activated transcription factor that is considered to be the primary mediator of inflammatory responses. In acute ischemic stroke, PPAR $\gamma$ is reported to be activated, which has been demonstrated to directly decrease tissue damage through inhibiting the NF- $\kappa B$ pathway, decreasing inflammation and stimulating the Nrf2/ARE axis to decrease oxidative stress (78). A previous study observed that 10-O-(N,N-dimethylaminoethyl)-ginkgolide B methanesulfonate, a novel derivative of Ginkgolide B, could transform polarized BV2 microglia from the M1 to the M2 phenotype through promoting PPAR $\gamma$ translocation from the nucleus to the cytoplasm following OGD; this subsequently promoted the decreased expression levels of TNF- $\alpha$ and the increased expression levels of TGF- $\beta 1$ (26). Additionally, IL-4 generated by neurons was identified to bind to the IL- 4 receptor expressed on the surface of microglia and activate M2 microglia through regulating the PPAR $\gamma$ signaling pathway (52); the activated M2 microglia were observed to phagocytose damaged cells and secrete brain-derived neurotrophic factor (BDNF) to decrease ischemic brain damage (52). Based on these studies, it was suggested that the modulation of PPAR $\gamma$ and its associated signaling pathways may represent a potential target for the treatment of ischemic stroke.

Receptors. Receptors are proteins, usually cell surface receptors, that recognize and bind to ligands and serve an important role in the signaling pathways involved in inducing local inflammation, the recruitment of new effector cells, the containment of local infection and the initiation of an adaptive immune response. Previous studies have identified that 3 important membrane receptors, TLR4, sphingosine 1 phosphate receptors (S1PRs) and thromboxane A2 receptor (TXA2R), are closely associated with microglia polarization $(31,70,79,80)$.

TLR4.TLR4, which is predominantly expressed by microglia, is an important regulator of inflammatory responses (79). Increased protein expression levels of TLR4 are reportedly associated with poor outcomes in patients with ischemic stroke (81). Furthermore, in ischemic stroke, TLR4 has been observed to recognize damage-associated molecules, such as lipopolysaccharide (LPS) and high mobility group box-1, and trigger inflammatory responses (Fig. 2) $(82,83)$. In mice, the number of TLR $4^{+}$cells, as well as $\mathrm{NF}^{-} \mathrm{KB}^{+}$and IL-1 $\beta^{+}$cells, was significantly increased at $72 \mathrm{~h}$ following MCAO; the number of TLR $4^{+}$cells was identified to be closely positively correlated with the number of IL- $1 \beta^{+}$cells and the infarct volume (84). In addition, in $T L R 4^{-/}$mice, the infarct volume was decreased compared with TLR4 ${ }^{+/+}$mice following MCAO (85). These results have also been validated in vitro; in BV2 microglia, a significant increase in TLR4 expression levels were detected following OGD, which was accompanied by an increase in proinflammatory cytokines, including TNF- $\alpha$, IL-1 $\beta$ and IL-6 (86); however, in TLR $4^{-1-}$ mice, expression levels of these cytokines generated by M1 microglia were observed to be significantly decreased through the inhibition of the MyD88/NF- $\mathrm{KB}$ signaling pathway $(79,87)$. These results suggested that the overexpression of TLR4 may activate and promote M1 microglia to trigger neuroinflammatory responses in ischemic stroke, which subsequently aggravates the brain damage. However, in another study, TLR4 was demonstrated to be positively associated with brain tissue repair (85): TLR4 activation regulated cell proliferation and neuroblast migration in the subventricular zone on days 1, 7 and 14 following MCAO, which suggested an effective means to modulate neurogenesis following ischemic stroke. Based on this conflicting evidence, further studies on the effects of TLR4 in different cells and at different time points in ischemic stroke are required.

SIPRs. S1PR1-S1PR5 are G-protein-coupled receptors that are expressed in abundance in the microglia and are demonstrated to regulate inflammatory responses following ischemic stroke (88). S1P is the ligand for S1PR and has been identified to bind to S1PR1, S1PR2 and S1PR3 to trigger neuroinflammatory responses in ischemic stroke (Fig. 2) (89-91). An in vitro study reported that upon the addition of S1P to the culture medium of primary microglia, the expression levels of IL-17 were increased (31). Additionally, the exogenous administration of S1P to OGDR microglia aggravated OGDR-induced neuronal apoptosis (31). In MCAO model mice, sphingosine kinase 1 (Sphk1) was demonstrated to phosphorylate sphingosine to S1P, and then S1P bound to S1PR3 to activate M1 microglia, which released proinflammatory cytokines and aggravated brain damage. This culminated in an increase in the expression levels of S1P and M1 microglial markers, CD11b, CD16, CD32, CD86, TNF- $\alpha$ and IL-1 $\beta$, as well as an increased infarct volume and neurological deficit (81). CAY10444, an inhibitor of S1PR3, and PF-543, an inhibitor of Sphk1, were identified to partially reverse the result to increase the expression levels (31,91); however, Qin et al (71) described opposing results, following the observation that Fingolimod, an S1PR agonist, inhibited M1 microglia-induced neuroinflammation and shift microglia toward M2 polarization through regulating STAT3 signaling in a BCAS-induced model of ischemic stroke. Therefore, additional studies are required to determine the role of S1P and S1PRs in the regulation of microglia polarization following ischemic stroke. 
$T X A 2 R$. TXA2R, another G-protein-coupled receptor, can promote platelet activation and aggregation, which regulates thrombosis/hemostasis and inflammatory responses $(92,93)$. In ischemia/reperfusion mice, significant TXA2R expression levels were detected in microglia/macrophages by immunofluorescence double staining (80). In addition, the expression levels of M1 microglia markers, such as CD16, CD86, IL-1 $\beta$, IL-6 and TNF- $\alpha$, were significantly increased in ischemic brains (80). However, SQ29548, a TXA2R antagonist, inhibited the activation of M1 microglia and subsequent inflammatory responses, which culminated in a decrease in CD16, CD86, IL-1 $\beta$, IL- 6 and TNF- $\alpha$ expression levels.

Ion channels. The expression of ion channels changes in response to voltage and $\mathrm{pH}$ gradients in the microenvironment, which induces intracellular signal transduction. Currently, 2 important ion channels, Hv1 and Kv1.3, have been reported to be closely associated with microglia polarization $(83,94)$.

$H v 1$. Hv1 is a voltage-gated proton channel that enables NADPH oxidase (NOX) to produce ROS by sensing both voltage and $\mathrm{pH}$ gradients (95). ROS have been identified to be one of the main factors contributing to the induction of M1 microglia to release proinflammatory cytokines (96). For example, a previous study demonstrated that an increase in Hv1 expression levels induced neuroinflammatory responses through the Hv1/NOX/ROS pathway, thereby exacerbating brain damage in ischemic stroke (95). Furthermore, in both an MCAO-induced and a PT-induced model of ischemic stroke, large Hv1-mediated currents were detected in microglia, but not in neurons or astrocytes $(94,95)$; this was accompanied by increased expression levels of ROS in the brain, which activated its downstream target $\mathrm{NF}-\kappa \mathrm{B}$ to trigger the secretion of proinflammatory cytokines generated by M1 microglia, further aggravating brain damage following ischemic stroke. In $H v I^{-/-}$mice, the brain infarct volume, neuronal damage and motor deficits were attenuated compared with wild-type mice following PT induction, which was accompanied by decreased expression levels of the M1 microglia markers, iNOS and CD16 (94). Additionally, the study also confirmed that more ROS could be released by M1 microglia following ischemic stroke, and in turn, these increased levels of ROS promoted M1 microglia polarization, resulting in a vicious circle of neuroinflammation, which exacerbated neuronal death. Another study demonstrated that the regenerative rate of oligodendrocyte precursor cells was increased, and subsequently the periventricular leukomalacia was reduced in $\mathrm{Hvl}^{-/-}$mice, confirming that Hv1 may aggravate brain damage (96). These results indicated that Hv1 may aggravate brain damage through increasing the expression levels of ROS and proinflammatory cytokines generated by M1 microglia; however, whether Hv1 affects M2 microglia polarization remains unclear.

$K v 1.3$. Previous studies have identified that changes in the expression levels of $\mathrm{K}^{+}$channels also occur in ischemic stroke and this can regulate microglia polarization $(83,97)$. $\mathrm{K}^{+}$channels are membrane proteins that permit the rapid and selective flow of $\mathrm{K}^{+}$ions across the cell membrane, thus generating electrical signals in cells. In response to brain ischemia, the expression levels of Kv1.3 were observed to be increased in microglia of both rodents and humans, which modulated $\mathrm{Ca}^{2+}$ signaling and induced neuroinflammation (97). In addition, in an LPS-induced microglia activated mouse model, M1 microglia were activated, accompanied by an increase in the current density and mRNA expression levels of $K v 1.3$ and inflammatory cytokines, such as TNF- $\alpha$, IL-1 $\beta$ and IL-6, in addition to iNOS (83). Notably, Kv1.3 knockout or treatment with the Kv1.3 inhibitor 5-(4-phenoxybutoxy)psoralen (PAP-1) could partially reverse these results; in LPS-activated microglia the expression levels of Kv1.3 and M1 microglia markers, such as TNF- $\alpha$, IL-1 $\beta$ and nitric oxide were significantly increased; however, PAP-1 was observed to decrease the polarization of M1 microglia, as well as the expression levels of proinflammatory cytokines (83). These data suggested that Kv1.3 may be one of the main mediators of M1 microglia polarization.

Modulators of gene expression. H19 imprinted maternally expressed transcript (H19), microRNA (miRNA)-155, miRNA-124 and chemokine-like protein TAFA-3 (FAM19A3) have been reported to be closely associated with inflammatory responses; the first 2 factors are closely associated with M1 polarization, whereas the latter 2 factors are associated with M2 polarization (18,98-100).

H19. H19 is a long non-coding RNA (lncRNA) that has been demonstrated to induce the onset of ischemic stroke and participate in the chronic regeneration process following ischemic stroke (98,101-103). In fact, H19 has been demonstrated to promote M1 microglia polarization and induce neuroinflammation in ischemic stroke through regulating histone deacetylases (HDACs) $(98,104,105)$. In clinical experiments $(98,101), H 19$ levels were identified to be significantly increased in the plasma at $3 \mathrm{~h}, 7,30$ and 90 days post-ischemic stroke, in addition to being positively correlated with the National Institute of Health Stroke Scale score (106) and TNF- $\alpha$ expression levels. Furthermore, in MCAO model mice, H19 expression levels were increased in both the plasma and the brain (98). Notably, H19 knockdown decreased the infarct volume and brain edema and decreased TNF- $\alpha$ and IL-1 $\beta$ expression levels in the brain following ischemic stroke (98). Furthermore, the study also demonstrated that $H 19$ knockdown could inhibit OGD-induced M1 microglia polarization in BV2 cells, which manifested as a decrease in the expression levels of TNF- $\alpha$ and CD11b (98). In addition, $H 19$ knockdown reversed the OGD-induced upregulation of HDAC1, whereas HDAC1 overexpression reversed the effects of $H 19$ knockdown and promoted microglia polarization toward the M1 phenotype (98). These data could be used to regulate M1 microglia polarization through the $H 19 / \mathrm{HDAC} 1$ signaling pathway.

miRNA-155. Increased expression levels of miRNA-155 have been demonstrated to be associated with the neuroinflammatory responses $(20,107)$. In LPS-activated microglia, miRNA-155 expression levels were significantly increased, which could target suppressor of cytokine signaling 1 to trigger M1 microglia-mediated inflammatory responses and aggravate brain damage (18). In addition, $m i R N A-155^{-/}$mice were reported to partially reverse LPS-induced neurological 
deficits and microglia activation (107); however, the roles of miRNA-155 in microglia polarization in ischemic stroke require additional clarification.

miRNA-124. miRNA-124 is the most abundant miRNA present in the CNS and is involved extensively in neuroprotective mechanisms $(108,109)$. In fact, it has been identified to regulate microglia activation and polarization in ischemic stroke $(99,110)$. A previous study demonstrated that miRNA-124 induced neuroprotection and functional improvement through regulating M2 microglia polarization in ischemic stroke (99); upon injection of miRNA-124 into the mouse ipsilateral hemisphere, the number of M2 microglia/macrophages was markedly increased, contributing to neuronal survival by releasing the trophic factor Arg-1 following MCAO. miRNA-124 treatment also resulted in the accelerated infiltration of reactive astrocytes into the lesion area, which subsequently resulted in a tighter glial scar border; however, whether the activation and polarization of microglia affects astrocyte function through modulating miRNA-124 remains unclear.

FAM19A3. FAM19A3, a member of the TAFA family of genes (TAFA1-5), is predominantly expressed in the CNS (111). Shao et al (100) suggested that FAM19A3 expression levels were significantly increased in the microglia of MCAO model mice, which promoted M2 microglia polarization and ameliorated cerebral ischemia. Notably, within these mice, FAM19A3 expression levels in the brain were elevated 1-3 days following MCAO, peaked at day 5, began to decrease at day 7 and returned to pre-injury levels by day 14, which is consistent with the pattern of M2 microglia markers, CD206, IL-10, Ym1/2 and TGF- $\beta$ discussed previously. Additionally, FAM19A3 was observed to be secreted by M2 microglia and in turn, FAM19A3 increased the expression levels of M2 microglia markers in a dose-dependent manner (100). These results suggested that FAM19A3 may be a genetic marker for M2 microglia; however, the underlying molecular mechanisms remains to be investigated.

Drug modulators. In clinical and experimental studies, a large number of drugs have been demonstrated to serve important roles as modulators of neuroinflammation and microglia polarization; for example, in both a PT-induced stroke model and BCAS-induced model, Fingolimod, a United States of America Food and Drug Administration-approved drug for the treatment of relapsing-remitting multiple sclerosis, modulated microglia toward M2 polarization, protected against ischemic damage and promoted angiogenesis through suppressing the STAT3 signaling pathway $(47,71)$. In MCAO model mice, rosiglitazone decreased the numbers of $\mathrm{Iba}^{+} / \mathrm{CD} 16^{+} \mathrm{M} 1$ microglia and increased the numbers of $\mathrm{Iba}^{+} / \mathrm{CD} 206^{+} \mathrm{M} 2$ microglia following stroke, which improved the overall long-term white matter integrity $(61,112)$. In addition to western medicines, some traditional Chinese medicines can also be used to regulate microglia polarization $(16,25,113)$; a study by Yang et al (16) demonstrated that baicalein significantly decreased the expression levels of the M1 markers, CD16 and CD86, and increased the expression levels of the M2 markers, CD163 and CD206. Additionally, expression levels of the proinflammatory factors,
IL-6, IL-18 and TNF- $\alpha$ were decreased upon inhibition of $\mathrm{NF}-\kappa \mathrm{B}$ signaling following baicalein treatment in MCAO model mice (16). Zhu et al (25) demonstrated that berberine could inhibit M1 polarization and promote M2 polarization to protect against ischemic stroke through the AMPK signaling pathway. Wang et al (46) reported that xinnao shutong capsules, a Chinese medicine produced in China, decreased the number of M1 microglia and increased the number of M2 microglia, which improved memory impairments in a BCCA-induced model of ischemic stroke (46). Nonetheless, despite the effects of these drugs on microglia polarization being confirmed in experimental studies, clinical studies are still lacking.

\section{Autophagy}

Autophagy is a cellular metabolic pathway through which damaged organelles and misfolded proteins are degraded and recycled to maintain cellular homeostasis. Accumulating evidence has reported that autophagy is activated in brain cells such as neurons, endothelial cells and microglia in ischemic stroke, and interfering with autophagy can aggravate brain damage (114-116). For example, in an in vitro study, the inhibition of autophagic flux with $\mathrm{NH}_{4} \mathrm{Cl}$ was demonstrated to aggravate the damage to brain cells, as it stimulated M1 microglia polarization and the release of TNF- $\alpha$, iNOS and cyclooxygenase-2 (117). In addition, the expression levels of M2 markers, including IL-10, Arg-1 and BDNF were decreased following the treatment of $\mathrm{NH}_{4} \mathrm{Cl}$ in OGD microglia. mTOR, a 289-kDa serine/threonine protein kinase, was demonstrated to inhibit autophagy through regulating its downstream signaling pathways (118), whereas Li et al (119) observed that mTOR complex 1 (mTORC1), a form of mTOR, prompted microglia to shift towards the M1 phenotype. Moreover, the inhibition of mTORC1 decreased the infarct volume and improved motor function, which lead to a decrease in the production of proinflammatory cytokines, including iNOS, TNF- $\alpha$ and IL-1 $\beta$, and chemokines, $\mathrm{C}-\mathrm{C}$ motif chemokine 2 and $\mathrm{C}-\mathrm{C}$ motif chemokine ligand 22, by M1 microglia. However, another previous study proposed the opposite view and suggested that autophagy may stimulate a microglial shift toward the M1 phenotype to exacerbate cerebral ischemia (120). In an in vitro study, the expression levels of the autophagy markers microtubule-associated proteins 1A/1B light chain 3B lipid modified form, beclin-1 and autophagy protein 5 were increased, which was accompanied by increased expression levels of the proinflammatory factors IL- $1 \beta, \mathrm{TNF}-\alpha$ and iNOS in primary microglia under OGD (120); however, these proinflammatory factors and the number of M1 microglia were downregulated by the autophagy inhibitor 3-methyladenine (3-MA). Moreover, 3-MA also prevented M2 microglia polarization through inhibiting the activation of CREB. Thus, based on these contradictory results, the roles of autophagy in microglia polarization in ischemic stroke require further investigation.

\section{Conclusions}

Microglia activation and polarization is considered to be important in brain damage and repair following ischemic stroke. The present review provided an overview of the activation and changes of M1 and M2 microglia following ischemic 
stroke. This is useful for determining the optimal time point at which to target microglia to treat ischemic stroke. Additionally, studies investigating the modulators of microglia activation and polarization were analyzed, as well as the role of autophagy in microglia polarization in ischemic stroke. This is of importance as it provides therapeutic targets to target microglia in ischemic stroke and it is also critical for our understanding of the pathophysiology and future investigations of ischemic stroke. It is worth noting that according to the American Heart Association/American Stroke Association guidelines intracerebral hemorrhage and ischemic stroke are 2 different types of stroke with different pathogenesis and treatments $(106,121)$. Lan et al (122) have already reviewed the modulation of microglial activation and polarization in intracerebral hemorrhage from 3 aspects: Microglial activation and function in intracerebral hemorrhage; modulators of microglial polarization; and microglial interactions with other cells. However, the present review differs from the review by Lan et al (122), as the present study investigated the changes and modulation of microglia in ischemic stroke. Additionally, Lan et al (122) primarily focused on the modulation of microglial polarization by the transcription factors and receptors. In addition to these typical modulation factors, which also serve a similar role in the modulation of microglia in ischemic stroke, the present review also discussed other factors associated with the modulation of microglial polarization, such as ion channels, genes and drugs. Notably, the time and spatial changes of microglia in ischemic stroke were further examined in this review. In practice, this review suggested future research directions to provide novel strategies for the treatment of ischemic stroke.

Based on the data presented in the present review, several issues can be selected for further investigation. Firstly, the experimental models of ischemic stroke need to be improved and more data on the experimental models needs to be collected and collated, to decrease the discrepancies reported between studies. Secondly, beneficial factors identified that regulated the polarization of microglia towards the M2 phenotype in animals with ischemic stroke require further investigations. Thirdly, further investigations are required into the role of autophagy in microglia polarization to improve our understanding of the pathology of ischemic stroke. In addition, regarding the extensive roles of microglia in ischemic stroke, investigations into the interaction between microglia and other cells will be important to identify the effective pathophysiological pathways that prevent brain damage. Finally, the focus of this review was on the pathophysiological regulatory mechanisms of microglia polarization. Therefore, the range of potential drug targets has not been fully explored. Our future studies will focus on other aspects of the roles of microglia in ischemia.

\section{Acknowledgements}

Not applicable.

\section{Funding}

This work was supported by the National Natural Science Foundation of China (grant nos. 81874416 and 81774174), the Hunan Education Department's Science \& Research Project (grant no. 16K063) and the Department of Integrated
Traditional Chinese and Western Medicine, Hunan University of Traditional Chinese Medicine (grant no. 2018ZXYJH01).

\section{Availability of data and materials}

Not applicable.

\section{Authors' contributions}

CTJ collected the literature, performed the analysis and interpretation and wrote the manuscript. WFW, YHD and JWG contributed to literature collection, and analysis and interpretation of the results. CTJ, YHD and JWG revised the manuscript. All authors reviewed the final draft of the manuscript.

\section{Ethics approval and consent to participate}

Not applicable.

\section{Patient consent for publication}

Not applicable.

\section{Competing interests}

The authors declare that they have no competing interests.

\section{References}

1. World Health Organization (WHO): Top 10 global causes of deaths, 2016. https://www.who.int/en/news-room/fact-sheets/detail/ the-top-10-causes-of-death. WHO, Geneva, 2016. Accessed May 24, 2018.

2. GBD 2016 Stroke Collaborators: Global, regional, and national burden of stroke, 1990-2016: A systematic analysis for the Global Burden of Disease Study 2016. Lancet Neurol 18: 439-458, 2019.

3. Feigin VL, Lawes CMM, Bennett DA, Barker-Collo SL and Parag V: Worldwide stroke incidence and early case fatality reported in 56 population-based studies: A systematic review. Lancet Neurol 8: 355-369, 2009.

4. Navis A, Garcia-Santibanez R and Skliut M: Epidemiology and outcomes of ischemic stroke and transient ischemic attack in the adult and geriatric population. J Stroke Cerebrovasc Dis 28: 84-89, 2019.

5. Ozaki T, Nakamura $\mathrm{H}$ and Kishima $\mathrm{H}$ : Therapeutic strategy against ischemic stroke with the concept of neurovascular unit. Neurochem Int 126: 246-251, 2019.

6. Thiebaut AM, Gauberti M, Ali C, Martinez De Lizarrondo S, Vivien D, Yepes M and Roussel BD: The role of plasminogen activators in stroke treatment: Fibrinolysis and beyond. Lancet Neurol 17: 1121-1132, 2018.

7. Xu XJ, Zhang L, Ye XC, Hao Q, Zhang T, Cui GY and Yu M: Nrf2/ARE pathway inhibits ROS-induced NLRP3 inflammasome activation in BV2 cells after cerebral ischemia reperfusion. Inflamm Res 67: 57-65, 2018.

8. Azedi F, Mehrpour M, Talebi S, Zendedel A, Kazemnejad S Mousavizadeh K, Beyer C, Zarnani A and Joghataei MT: Melatonin regulates neuroinflammation ischemic stroke damage through interactions with microglia in reperfusion phase. Brain Res 1723: 146401, 2019

9. Zhuang P, Wan Y, Geng S, He Y, Feng B, Ye Z, Zhou D, Li D, Wei H, $\mathrm{LiH}$, et al: Salvianolic Acids for Injection (SAFI) suppresses inflammatory responses in activated microglia to attenuate brain damage in focal cerebral ischemia. J Ethnopharmacol 198: 194-204, 2017.

10. Chamorro Á, Dirnagl U, Urra X and Planas AM: Neuroprotection in acute stroke: Targeting excitotoxicity, oxidative and nitrosative stress, and inflammation. Lancet Neurol 15: 869-881, 2016.

11. Zhou SJ, Zhu WQ, Zhang Y,Pan SP and Bao JH: S100B promotes microglia M1 polarization and migration to aggravate cerebral ischemia. Inflamm Res 67: 937-949, 2018. 
12. Zhao HR, Wan LH, Chen Y, Zhang H, Xu Y and Qiu SW: FasL incapacitation alleviates CD4(+) T cells-induced brain injury through remodeling of microglia polarization in mouse ischemic stroke. J Neuroimmunol 318: 36-44, 2018.

13. Xiong XY, Liu L and Yang QW: Functions and mechanisms of microglia/macrophages in neuroinflammation and neurogenesis after stroke. Prog Neurobiol 142: 23-44, 2016.

14. Neher JJ and Cunningham C: Priming microglia for innate immune memory in the brain. Trends Immunol 40: 358-374, 2019.

15. Li B, Concepcion K, Meng XM and Zhang LB: Brain-immune interactions in perinatal hypoxic-ischemic brain injury. Prog Neurobiol 159: 50-68, 2017.

16. Yang S, Wang H, Yang Y, Wang R, Wang Y, Wu C and Du G: Baicalein administered in the subacute phase ameliorates ischemia-reperfusion-induced brain injury by reducing neuroinflammation and neuronal damage. Biomed Pharmacother 117: 109102, 2019.

17. Weng L, Wu Z, Zheng W, Meng H, Han L, Wang S, Yuan Z and $\mathrm{Xu}$ Y: Malibatol A enhances alternative activation of microglia by inhibiting phosphorylation of Mammalian Ste20-like kinase1 in OGD-BV-2 cells. Neurol Res 38: 342-348, 2016.

18. Zheng X, Huang H, Liu J, Li M, Liu M and Luo T: Propofol attenuates inflammatory response in LPS-activated microglia by regulating the miR-155/SOCS1 pathway. Inflammation 41: 11-19, 2018.

19. Meng HL, Li XX, Chen YT, Yu LJ, Zhang H, Lao JM, Zhang X and $\mathrm{Xu}$ Y: Neuronal soluble Fas ligand drives M1-microglia polarization after cerebral ischemia. CNS Neurosci Ther 22: 771-781, 2016

20. Greco R, Demartini C, Zanaboni AM, Blandini F, Amantea D and Tassorelli C: Endothelial nitric oxide synthase inhibition triggers inflammatory responses in the brain of male rats exposed to ischemia-reperfusion injury. J Neurosci Res 96 : 151-159, 2018.

21. Ji J, Xiang P, Li T, Lan L, Xu X, Lu G, Ji H, Zhang Y and Li Y: NOSH-NBP, a novel nitric oxide and hydrogen sulfide- releasing hybrid, attenuates ischemic stroke-induced neuroinflammatory injury by modulating microglia polarization. Front Cell Neurosci 11: 154, 2017

22. Wang J, Xing H, Wan L, Jiang X, Wang $C$ and Wu Y: Treatment targets for M2 microglia polarization in ischemic stroke. Biomed Pharmacother 105: 518-525, 2018.

23. Liu X, Liu J, Zhao S, Zhang H, Cai W, Cai M, Ji X, Leak R, Gao Y, Chen $\mathrm{J}$ and $\mathrm{Hu} \mathrm{X}$ : Interleukin-4 is essential for microglia/macrophage M2 polarization and long-term recovery after cerebral ischemia. Stroke 47: 498-504, 2016.

24. Feng YK, He XF, Luo SJ, Chen XF, Long SM, Liang FY, Shi TT, Pei Z and Li ZD: Chronic colitis induces meninges traffic of gut-derived T cells, unbalances M1 and M2 microglia/macrophage and increases ischemic brain injury in mice. Brain Res 1707: 8-17, 2019.

25. Zhu J, Cao D, Guo C, Liu M, Tao Y, Zhou J, Wang F, Zhao Y, Wei J, Zhang Y, et al: Berberine facilitates angiogenesis against ischemic stroke through modulating microglial polarization via AMPK signaling. Cell Mol Neurobiol 39: 751-768, 2019.

26. Liu R, Diao J, He S, Li B, Fei Y, Li Y and Fang W: XQ-1H protects against ischemic stroke by regulating microglia polarization through PPARgamma pathway in mice. Int Immunopharmacol 57: 72-81, 2018.

27. Boddaert J, Bielen K, 's Jongers B, Manocha E, Yperzeele L, Cras P, Pirici D and Kumar-Singh S: CD8 signaling in microglia/macrophage M1 polarization in a rat model of cerebral ischemia. PLoS One 13: e0186937, 2018.

28. Collmann FM, Pijnenburg R, Hamzei-Taj S, Minassian A, Folz-Donahue K, Kukat C, Aswendt M and Hoehn M: Individual in vivo profiles of microglia polarization after stroke, represented by the genes iNOS and Ym1. Front Immunol 10: 1236, 2019.

29. Chen AQ, Fang Z, Chen XL, Yang S, Zhou YF, Mao L, Xia YP, Jin HJ, Li YN, You MF, et al: Microglia-derived TNF-alpha mediates endothelial necroptosis aggravating blood brain-barrier disruption after ischemic stroke. Cell Death Dis 10: 487, 2019.

30. Moraes CA, Santos G, de Sampaio e Spohr TC, D'Avila JC, Lima FR, Benjamim CF, Bozza FA and Gomes FC: Activated microglia-induced deficits in excitatory synapses through IL-1 $\beta$ : Implications for cognitive impairment in sepsis. Mol Neurobiol 52: 653-663, 2015.

31. Lv M, Zhang D, Dai D, Zhang W and Zhang L: Sphingosine kinase $1 /$ sphingosine-1-phosphate regulates the expression of interleukin-17A in activated microglia in cerebral ischemia/reperfusion. Inflamm Res 65: 551-562, 2016.
32. Xie L, Sun F, Wang J, Mao X, Xie L, Yang S, Su D, Simpkins J, Greenberg D and Jin K: mTOR signaling inhibition modulates macrophage/microglia-mediated neuroinflammation and secondary injury via regulatory $\mathrm{T}$ cells after focal ischemia. J Immunol 192: 6009-6019, 2014.

33. Wang SW, Zhang $\mathrm{H}$ and $\mathrm{Xu}$ Y: Crosstalk between microglia and $T$ cells contributes to brain damage and recovery after ischemic stroke. Neurol Res 38: 495-503, 2016.

34. Zhou T, Huang Z, Sun X, Zhu X, Zhou L, Li M, Cheng B, Liu X and He C: Microglia polarization with M1/M2 phenotype changes in rd1 mouse model of retinal degeneration. Front Neuroanat 11: 77, 2017.

35. Huang M, Li Y, Wu K, Yan W, Tian T, Wang Y and Yang H: Paraquat modulates microglia M1/M2 polarization via activation of TLR4-mediated NF- $\kappa \mathrm{B}$ signaling pathway. Chem Biol Interact 310: 108743, 2019.

36. Huang XP, Peng JH, Pang JW, Tian XC, Li XS, Wu Y, Li Y, Jiang Y and Sun XC: Peli1 contributions in microglial activation, neuroinflammatory responses and neurological deficits following experimental subarachnoid hemorrhage. Front Mol Neurosci 10: 398, 2017.

37. Liu LQ, Liu XR, Zhao JY, Yan F, Wang RL, Wen SH, Wang L, Luo YM and Ji XM: Brain-selective mild hypothermia promotes long-term white matter integrity after ischemic stroke in mice. CNS Neurosci Ther 24: 1275-1285, 2018.

38. Jiang M, Liu X, Zhang D, Wang Y, Hu X, Xu F, Jin M, $\mathrm{Cao} \mathrm{F}$ and $\mathrm{Xu} \mathrm{L}$ : Celastrol treatment protects against acute ischemic stroke-induced brain injury by promoting an IL-33/ST2 axis-mediated microglia/macrophage M2 polarization. J Neuroinflammation 15: 78, 2018.

39. Jin Q, Cheng J, Liu Y, Wu J, Wang X, Wei S, Zhou X, Qin Z, Jia $\mathrm{J}$ and $Z$ hen $\mathrm{X}$ : Improvement of functional recovery by chronic metformin treatment is associated with enhanced alternative activation of microglia/macrophages and increased angiogenesis and neurogenesis following experimental stroke. Brain Behav Immun 40: 131-142, 2014

40. Li R, Liu WC, Yin J, Chen YC, Guo SQ, Fan HY, Li XF, Zhang X, He XY and Duan CZ: TSG-6 attenuates inflammation-induced brain injury via modulation of microglial polarization in SAH rats through the SOCS3/STAT3 pathway. J Neuroinflammation 15: 231,2018

41. Liu Z, Ran Y, Huang S, Wen S, Zhang W, Liu X, Ji Z, Geng X, $\mathrm{Ji} \mathrm{X}, \mathrm{Du} \mathrm{H}$, et al: Curcumin protects against ischemic stroke by titrating microglia/macrophage polarization. Front Aging Neurosci 9: 233, 2017

42. Satoh J, Kino Y, Asahina N, Takitani M, Miyoshi J, Ishida T and Saito Y: TMEM119 marks a subset of microglia in the human brain. Neuropathology 36: 39-49, 2016.

43. Huang M, Wan Y, Mao L, He QW, Xia YP, Li M, Li YN, Jin HJ and Hu B: Inhibiting the migration of M1 microglia at hyperacute period could improve outcome of tMCAO rats. CNS Neurosci Ther 23: 222-232, 2017

44. Hu X, Li P, Guo Y, Wang H, Leak R, Chen S, Gao Y and Chen J: Microglia/macrophage polarization dynamics reveal novel mechanism of injury expansion after focal cerebral ischemia. Stroke 43: 3063-3070, 2012.

45. Hwang IK, Park JH, Lee TK, Kim DW, Yoo KY, Ahn JH, Kim YH, Cho JH, Kim YM, Won MH and Moon SM: CD74-immunoreactive activated M1 microglia are shown late in the gerbil hippocampal CA1 region following transient cerebral ischemia. Mol Med Rep 15: 4148-4154, 2017.

46. Wang L, Wang R, Chen Z, Zhao H and Luo Y: Xinnao Shutong modulates the neuronal plasticity through regulation of microglia/macrophage polarization following chronic cerebral hypoperfusion in rats. Front Physiol 9: 529, 2018.

47. Shang K, He J, Zou J, Qin C, Lin L, Zhou LQ, Yang LL, Wu LJ, Wang W, Zhan KB and Tian DS: Fingolimod promotes angiogenesis and attenuates ischemic brain damage via modulating microglial polarization. Brain Res 1726: 146509, 2020.

48. Yang L, Tucker D, Dong Y, Wu C, Lu Y, Li Y, Zhang J, Liu TC and Zhang Q: Photobiomodulation therapy promotes neurogenesis by improving post-stroke local microenvironment and stimulating neuroprogenitor cells. Exp Neurol 299: 86-96, 2017.

49. Zong X, Dong Y, Li Y, Yang L, Li Y, Yang B, Tucker L, Zhao N, Brann DW, Yan X, et al: Beneficial effects of theta-burst transcranial magnetic stimulation on stroke injury via improving neuronal microenvironment and mitochondrial integrity. Transl Stroke Res: Sep 12, 2019 (Epub ahead of print). 
50. Zhao SC, Wang C, Xu H, Wu WQ, Chu ZH, Ma LS, Zhang YD and Liu F: Age-related differences in interferon regulatory factor-4 and -5 signaling in ischemic brains of mice. Acta Pharmacol Sin 38: 1425-1434, 2017.

51. Suenaga J, Hu X, Pu H, Shi Y, Hassan SH, Xu M, Leak RK, Stetler RA, Gao Y and Chen J: White matter injury and microglia/macrophage polarization are strongly linked with age-related long-term deficits in neurological function after stroke. Exp Neurol 272: 109-119, 2015.

52. Zhao X, Wang H, Sun G, Zhang J, Edwards NJ and Aronowski J: Neuronal interleukin-4 as a modulator of microglial pathways and ischemic brain damage. J Neurosci 35: 11281-11291, 2015.

53. Choi JY, Kim JY, Kim JY, Park J, Lee WT and Lee JE: M2 phenotype microglia-derived cytokine stimulates proliferation and neuronal differentiation of endogenous stem cells in ischemic brain. Exp Neurobiol 26: 33-41, 2017.

54. Perego C, Fumagalli S, Zanier ER, Carlino E, Panini N, Erba E and De Simoni MG: Macrophages are essential for maintaining a M2 protective response early after ischemic brain injury. Neurobiol Dis 96: 284-293, 2016.

55. Zheng Y, He RY, Wang P, Shi YJ, Zhao L and Liang J: Exosomes from LPS-stimulated macrophages induce neuroprotection and functional improvement after ischemic stroke by modulating microglial polarization. Biomater Sci 7: 2037-2049, 2019.

56. Zhang Q, Bian G, Chen P, Liu L, Yu C, Liu F, Xue Q, Chung SK, Song $\mathrm{B}, \mathrm{Ju} \mathrm{G}$ and Wang $\mathrm{J}$ : Aldose reductase regulates microglia/macrophages polarization through the cAMP response element-binding protein after spinal cord injury in mice. Mol Neurobiol 53: 662-676, 2016.

57. Li L, Yihao T, Zhou F, Yin N, Qiang T, Haowen Z, Qianwei C, Jun T, Yuan Z, Gang Z, et al: Inflammatory regulation by driving microglial M2 polarization: Neuroprotective effects of cannabinoid receptor- 2 activation in intracerebral hemorrhage. Front Immunol 8: 112, 2017.

58. Zhang Y, Xu N, Ding Y, Zhang Y, Li Q, Flores J, Haghighiabyaneh M, Doycheva D, Tang J and Zhang JH: Chemerin suppresses neuroinflammation and improves neurological recovery via CaMKK2/AMPK/Nrf2 pathway after germinal matrix hemorrhage in neonatal rats. Brain Behav Immun 70: 179-193, 2018

59. Shu ZM, Shu XD, Li HQ, Sun Y, Shan H, Sun XY, Du RH, Lu M, Xiao M, Ding JH and Hu G: Ginkgolide B protects against ischemic stroke via modulating microglia polarization in mice. CNS Neurosci Ther 22: 729-739, 2016.

60. Moretti R, Leger PL, Besson VC, Csaba Z, Pansiot J Di Criscio L, Gentili A, Titomanlio L, Bonnin P, Baud O and Charriaut-Marlangue C: Sildenafil, a cyclic GMP phosphodiesterase inhibitor, induces microglial modulation after focal ischemia in the neonatal mouse brain. J Neuroinflammation 13: 95, 2016.

61. Han L, Cai W, Mao L, Liu J, Li P, Leak RK, Xu Y, Hu X and Chen J: Rosiglitazone promotes white matter integrity and long-term functional recovery after focal cerebral ischemia. Stroke 46: 2628-2636, 2015.

62. Xiang B, Xiao C, Shen T and Li XF: Anti-inflammatory effects of anisalcohol on lipopolysaccharide-stimulated BV2 microglia via selective modulation of microglia polarization and down-regulation of NF-kappaB p65 and JNK activation. Mol Immunol 95: 39-46, 2018.

63. Chen J, Yin W, Tu Y, Wang S, Yang X, Chen Q, Zhang X, Han Y and Pi R: L-F001, a novel multifunctional ROCK inhibitor, suppresses neuroinflammation in vitro and in vivo: Involvement of NF- $\kappa \mathrm{B}$ inhibition and $\mathrm{Nrf} 2$ pathway activation. Eur J Pharmacol 806: 1-9, 2017.

64. Xiao L, Sun W, Lan W, Xiong Y, Duan Z, Zhang Z, Fan W, Xu L, Xie X, Ma N, et al: Correlation between cerebral microbleeds and S100B/RAGE in acute lacunar stroke patients. J Neurol Sci 340: 208-212, 2014.

65. Chen C, Chu SF, Ai QD, Zhang Z, Guan FF, Wang SS, Dong YX, Zhu J, Jian WX and Chen NH: CKLF1 Aggravates focal cerebral ischemia injury at early stage partly by modulating microglia/macrophage toward M1 polarization through CCR4. Cell Mol Neurobiol 39: 651-669, 2019.

66. Chen C, Ai Q, Chu S, Zhang Z, Zhou X, Luo P, Liu Y and Chen N: IMM-H004 protects against oxygen-glucose deprivation/reperfusion injury to BV2 microglia partly by modulating CKLF1 involved in microglia polarization. Int Immunopharmacol 70: 69-79, 2019.
67. Kong LL, Wang ZY, Hu JF, Yuan YH and Chen NH: Inhibition of chemokine-like factor 1 improves blood-brain barrier dysfunction in rats following focal cerebral ischemia. Neurosci Lett 627: 192-198, 2016

68. Kwon YW, Cheon SY, Park SY, Song J and Lee JH: Tryptanthrin suppresses the activation of the LPS-treated BV2 microglial cell line via Nrf2/HO-1 antioxidant signaling. Front Cell Neurosci 11: $18,2017$.

69. Liu R, Liao XY, Pan MX, Tang JC, Chen SF, Zhang Y, Lu PX, Lu LJ, Zou YY, Qin XP, et al: Glycine exhibits neuroprotective effects in ischemic stroke in rats through the inhibition of $\mathrm{M} 1$ microglial polarization via the NF- $\kappa \mathrm{B}$ p65/Hif-1 $\alpha$ signaling pathway. J Immunol 202: 1704-1714, 2019.

70. Butturini E, Boriero D, Carcereri de Prati A and Mariotto S: STAT1 drives M1 microglia activation and neuroinflammation under hypoxia. Arch Biochem Biophys 669: 22-30, 2019.

71. Qin C, Fan WH, Liu Q, Shang K, Murugan M, Wu LJ, Wang W and Tian DS: Fingolimod protects against ischemic white matter damage by modulating microglia toward M2 polarization via STAT3 pathway. Stroke 48: 3336-3346, 2017.

72. Ding Y, Qian J, Li H, Shen H, Li X, Kong Y, Xu Z and Chen G: Effects of SC99 on cerebral ischemia-perfusion injury in rats: Selective modulation of microglia polarization to M2 phenotype via inhibiting JAK2-STAT3 pathway. Neurosci Res 142: 58-68, 2019.

73. Zhou K, Chen J, Wu J, Wu Q, Jia C, Xu YXZ, Chen L, Tu W, Yang G, Kong J, et al: Atractylenolide III ameliorates cerebral ischemic injury and neuroinflammation associated with inhibiting JAK2/STAT3/Drp1-dependent mitochondrial fission in microglia. Phytomedicine 59: 152922, 2019.

74. Yang X, Xu S, Qian Y and Xiao Q: Resveratrol regulates microglia M1/M2 polarization via PGC-1alpha in conditions of neuroinflammatory injury. Brain Behav Immun 64: 162-172, 2017.

75. Zhang W, Wei R, Zhang L, Tan Y and Qian C: Sirtuin 6 protects the brain from cerebral ischemia/reperfusion injury through NRF2 activation. Neuroscience 366: 95-104, 2017.

76. Xue F, Huang JW, Ding PY, Zang HG, Kou ZJ, Li T, Fan J, Peng ZW and Yan WJ: Nrf2/antioxidant defense pathway is involved in the neuroprotective effects of Sirt1 against focal cerebral ischemia in rats after hyperbaric oxygen preconditioning. Behav Brain Res 309: 1-8, 2016.

77. Alfieri A, Srivastava S, Siow RC, Modo M, Fraser PA and Mann GE: Targeting the Nrf2-Keap1 antioxidant defence pathway for neurovascular protection in stroke. J Physiol 589: 4125-4136, 2011.

78. Cai W, Yang T, Liu H, Han LJ, Zhang K, Hu XM, Zhang XJ, Yin KJ, Cao YQ, Bennett MVL, et al: Peroxisome proliferator-activated receptor $\gamma$ (ppar $\gamma$ ): A master gatekeeper in CNS injury and repair. Prog Neurobiol 163-164: 27-28, 2018.

79. Yao X, Liu S, Ding W, Yue P, Jiang Q, Zhao M, Hu F and Zhang H: TLR4 signal ablation attenuated neurological deficits by regulating microglial M1/M2 phenotype after traumatic brain injury in mice. J Neuroimmunol 310: 38-45, 2017.

80. Yan A, Zhang T, Yang X, Shao J, Fu N, Shen FX, Fu Y and Xia W: Thromboxane A2 receptor antagonist SQ29548 reduces ischemic stroke-induced microglia/macrophages activation and enrichment, and ameliorates brain injury. Sci Rep 6: 35885, 2016.

81. Hyakkoku K, Hamanaka J, Tsuruma K, himazawa M, Tanaka H, Uematsu S, Akira S, Inagaki N, Nagai H and Hara H: Toll-like receptor 4 (TLR4), but not TLR3 or TLR9, knock-out mice have neuroprotective effects against focal cerebral ischemia. Neuroscience 171: 258-267, 2010.

82. Le K, Chibaatar Daliv E, Wu SS, Qian FY, Ali AI, Yu DF and Guo Y: SIRT1-regulated HMGB1 release is partially involved in TLR4 signal transduction: A possible anti-neuroinflammatory mechanism of resveratrol in neonatal hypoxic-ischemic brain injury. Int Immunopharmacol 75: 105779, 2019.

83. Di Lucente J, Nguyen HM, Wulff H, Jin LW and Maezawa I: The voltage-gated potassium channel Kv1.3 is required for microglial pro-inflammatory activation in vivo. Glia 66: 1881-1895, 2018.

84. Zhao Y, Wang H, Chen W, Chen L, Liu D, Wang X and Wang X: Melatonin attenuates white matter damage after focal brain ischemia in rats by regulating the TLR4/NF-kappaB pathway. Brain Res Bull 150: 168-178, 2019.

85. Palma-Tortosa S, Hurtado O, Pradillo JM, Ferreras-Martin R, García-Yébenes I, García-Culebras A, Moraga A, Moro MA and Lizasoain I: Toll-like receptor 4 regulates subventricular zone proliferation and neuroblast migration after experimental stroke. Brain Behav Immun 80: 573-582, 2019. 
86. Xiang HF, Cao DH, Yang YQ, Wang HQ, Zhu LJ, Ruan BH Du J and Wang MC: Isoflurane protects against injury caused by deprivation of oxygen and glucose in microglia through regulation of the Toll-like receptor 4 pathway. J Mol Neurosci 54 664-670, 2014.

87. Luo Y, Wang C, Li WH, Liu J, He HH, Long JH, Yang J, Sui X, Wang S, You Z and Wang YA: Madecassoside protects BV2 microglial cells from oxygen-glucose deprivation/reperfusion-induced injury via inhibition of the toll-like receptor 4 signaling pathway. Brain Res 1679: 144-154, 2018.

88. Spiegel S and Milstien S: Sphingosine-1-phosphate: An enigmatic signalling lipid. Nat Rev Mol Cell Biol 4: 397-407, 2003.

89. Gaire BP, Lee CH, Sapkota A, Lee SY, Chun J, Cho HJ, Nam TG and Choi JW: Identification of Sphingosine 1-Phosphate Receptor Subtype $1\left(\mathrm{~S}_{1} \mathrm{P}_{1}\right)$ as a pathogenic factor in transient focal cerebral ischemia. Mol Neurobiol 55: 2320-2332, 2018.

90. Kim GS, Yang L, Zhang G, Zhao H, Selim M, McCullough LD, Kluk MJ and Sanchez T. Critical role of sphingosine-1-phosphate receptor-2 in the disruption of cerebrovascular integrity in experimental stroke. Nat Commun 6: 7893, 2015.

91. Gaire BP, Song MR and Choi JW: Sphingosine 1-phosphate receptor subtype $3\left(\mathrm{~S}_{1} \mathrm{P}_{3}\right)$ contributes to brain injury after transient focal cerebral ischemia via modulating microglia activation and their M1 polarization. J Neuroinflammation 15 : 284,2018 .

92. Chen H: Role of thromboxane A2 signaling in endothelium-dependent contractions of arteries. Prostaglandins Other Lipid Mediat 134: 32-37, 2018.

93. Huang JS, Ramamurthy SK, Lin X and Breton GCL: Cell signalling through thromboxane A2 receptors. Cell Signal 16: 521-533, 2004

94.Tian DS, Li CY, Qin C, Murugan M, Wu LJ and Liu JL: Deficiency in the voltage-gated proton channel Hvl increases M2 polarization of microglia and attenuates brain damage from photothrombotic ischemic stroke. J Neurochem 139: 96-105, 2016.

95. Wu LJ, Wu G, Akhavan Sharif MR, Baker A, Jia Y, Fahey FH, Luo HR, Feener EP and Clapham DE: The voltage-gated proton channel Hv1 enhances brain damage from ischemic stroke. Nat Neurosci 15: 565-573, 2012.

96. Yu Y, Yu ZY, Xie MJ, Wang W and Luo X: Hv1 proton channel facilitates production of ROS and pro-inflammatory cytokines in microglia and enhances oligodendrocyte progenitor cells damage from oxygen-glucose deprivation in vitro. Biochem Biophys Res Commun 498: 1-8, 2018.

97. Nguyen HM, Grössinger EM, Horiuchi M, Davis KW, Jin LW, Maezawa I and Wulff H: Differential Kv1.3, KCa3.1, and Kir2.1 expression in 'classically' and 'alternatively' activated microglia. Glia 65: 106-121, 2017

98. Wang J, Zhao H, Fan Z, Li G, Ma Q, Tao Z, Wang R, Feng J and Luo Y: Long noncoding RNA H19 promotes neuroinflammation in ischemic stroke by driving histone deacetylase 1-dependent M1 microglial polarization. Stroke 48: 2211-2221, 2017.

99. Hamzei Taj S, Kho W, Riou A, Wiedermann D and Hoehn M MiRNA-124 induces neuroprotection and functional improvement after focal cerebral ischemia. Biomaterials 91: 151-165, 2016.

100. Shao Y, Deng T, Zhang T, Li P and Wang Y: FAM19A3, a novel secreted protein, modulates the microglia/macrophage polarization dynamics and ameliorates cerebral ischemia. FEBS Lett 589: 467-475, 2015

101. Wang J, Cao B, Zhao H, Gao Y, Luo Y, Chen Y and Feng J: Long noncoding RNA H19 prevents neurogenesis in ischemic stroke through p53/Notch1 pathway. Brain Res Bull 150: 111-117, 2019

102. Huang Y, Wang L, Mao Y and Nan G: Long noncoding RNA-H19 contributes to atherosclerosis and induces ischemic stroke via the upregulation of acid phosphatase 5. Front Neurol 10: 32, 2019.

103. Wang J, Cao B, Han D, Sun M and Feng J: Long non-coding RNA H19 induces cerebral ischemia reperfusion injury via activation of autophagy. Aging Dis 8: 71-84, 2017.

104. Huang Y, Zheng Y, Jin C, Li X, Jia L and Li W: Long non-coding RNA H19 inhibits adipocyte differentiation of bone marrow mesenchymal stem cells through epigenetic modulation of histone deacetylases. Sci Rep 6: 28897, 2016.

105. Han CL, Ge M, Liu YP, Zhao XM, Wang KL, Chen N, Meng WJ, $\mathrm{Hu}$ W, Zhang JG, Li L and Meng FG: LncRNA H19 contributes to hippocampal glial cell activation via JAK/STAT signaling in a rat model of temporal lobe epilepsy. J Neuroinflammation 15 : $103,2018$.
106. William JP, Alejandro AR, Teri A, Opeolu MA, Nicholas CB Kyra B, José B, Michael B, Bart MD, Brian H, et al: 2018 Guidelines for the early management of patients with acute ischemic stroke. Stroke 49: e46-e99, 2018.

107. Woodbury ME, Freilich RW, Cheng CJ, Asai H, Ikezu S, Boucher JD, Slack F and Ikezu T: miR-155 is essential for inflammation-induced hippocampal neurogenic dysfunction. J Neurosci 35: 9764-9781, 2015.

108. Sun Y, Luo ZM, Guo XM, Su DF and Liu X: An updated role of microRNA-124 in central nervous system disorders: A review. Front Cell Neurosci 9: 193, 2015.

109. Zhang W and Meng A: MicroRNA-124 expression in the brains of rats during early cerebral ischemia and reperfusion injury is associated with cell apoptosis involving STAT3. Exp Ther Med 17: 2870-2876, 2019.

110. Hamzei Taj S, Kho W, Aswendt M, Collmann FM, Green C, Adamczak J, Tennstaedt A and Hoehn M: Dynamic modulation of microglia/macrophage polarization by miR-124 after focal cerebral ischemia. J Neuroimmune Pharmacol 11: 733-748, 2016.

111. Tom Tang Y, Emtage P, Funk WD, Hu T, Arterburn M, Park EE and Rupp F: TAFA: A novel secreted family with conserved cysteine residues and restricted expression in the brain. Genomics 83: 727-734, 2004.

112. Wen L, You W, Wang H, Meng Y, Feng J and Yang X: Polarization of microglia to the M2 phenotype in a peroxisome proliferator-activated receptor gamma-dependent manner attenuates axonal injury induced by traumatic brain injury in mice. J Neurotrauma 35: 2330-2340, 2018.

113. Liu X, Wen S, Yan F, Liu K, Liu L, Wang L, Zhao S and Ji X: Salidroside provides neuroprotection by modulating microglial polarization after cerebral ischemia. J Neuroinflammation 15: 39, 2018.

114. Wang P, Shao BZ, Deng ZQ, Chen S, Yue ZY and Miao CY: Autophagy in ischemic stroke. Prog Neurobiol 163-164: 98-117, 2018.

115. Yang Z, Zhong L, Zhong S, Xian R and Yuan B: Hypoxia induces microglia autophagy and neural inflammation injury in focal cerebral ischemia model. Exp Mol Pathol 98: 219-224, 2015.

116. He HY, Ren L, Guo T and Deng YH: Neuronal autophagy aggravates microglial inflammatory injury by downregulating CX3CL1/fractalkine after ischemic stroke. Neural Regen Res 14: 280-288, 2019.

117. Xia CY, Zhang S, Chu SF, Wang ZZ, Song XY, Zuo W, Gao Y, Yang PF and Chen NH: Autophagic flux regulates microglial phenotype according to the time of oxygen-glucose deprivation/reperfusion. Int Immunopharmacol 39: 140-148, 2016.

118. Jiang J, Dai JC and Cui H: Vitexin reverses the autophagy dysfunction to attenuate MCAO-induced cerebral ischemic stroke via mTOR/Ulk1 pathway. Biomed Pharmacother 99 583-590, 2018

119. Li D, Wang C, Yao Y, Chen L, Liu G, Zhang R, Liu Q, Shi F and Hao J: mTORC1 pathway disruption ameliorates brain inflammation following stroke via a shift in microglia phenotype from M1 type to M2 type. FASEB J 30: 3388-3399, 2016

120. Jiang M, Wang H, Jin M, Yang X, Ji H, Jiang Y, Zhang H, Wu F, Wu G, Lai X, et al: Exosomes from mir-30d-5p-adscs reverse acute ischemic stroke-induced, autophagy-mediated brain injury by promoting $\mathrm{m} 2 \mathrm{microg} / \mathrm{ial} / \mathrm{macrophage}$ polarization. Cell Physiol Biochem 47: 864-878, 2018

121. Hemphill JC III, Greenberg SM, Anderson CS, Becker K, Bendok BR, Cushman M, Fung GL, Goldstein JN, Macdonald RL, Mitchell PH, et al: Guidelines for the management of spontaneous intracerebral hemorrhage. A Guideline for Healthcare Professionals From the American Heart Association/American Stroke Association. Stroke 46: 2032-2060, 2015.

122. Lan X, Han X, Li Q, Yang QW and Wang J: Modulators of microglial activation and polarization after intracerebral haemorrhage. Nat Rev Neurol 13: 420-433, 2017.

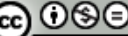

This work is licensed under a Creative Common Attribution-NonCommercial-NoDerivatives 4.0 International (CC BY-NC-ND 4.0) License. 